\title{
Hadronic off-shell width of meson resonances
}

\author{
D. Gómez Dumm \\ Departamento de Física, Comisión Nacional de Energía Atómica, Av. Libertador 8250, (1429) Buenos Aires, Argentina
}

A. Pich and J. Portolés

Departament de Física Teòrica, IFIC, CSIC, Universitat de València Edifici d'Instituts d'Investigació, Apt. Correus 2085, E-46071 València, Spain

(Received 3 April 2000; published 31 July 2000)

\begin{abstract}
Within the resonance chiral effective theory we study the dressed propagators of the spin-1 fields, which arise from a Dyson-Schwinger resummation perturbatively constructed from loop diagrams with absorptive contributions in the $s$ channel. We apply the procedure to the vector pion form factor and elastic $\pi \pi$ scattering to obtain the off-shell width of the $\rho^{0}$ meson. We adopt a definition of the off-shell width of spin- 1 meson resonances that satisfies the requirements of analyticity, unitarity, chiral symmetry, and asymptotic behavior ruled by QCD. To satisfy these constraints the resummation procedure cannot consist only of self-energy diagrams. Our width definition is shown to be independent of the formulation used to describe the spin-1 meson resonances.

PACS number(s): 12.39.Fe, 12.38.Aw, 12.38.Cy, 12.40.Vv
\end{abstract}

\section{INTRODUCTION}

The evaluation of hadronic current matrix elements in the low-energy regime is a long-standing problem of particle physics that has been addressed using many different tools. The common lore amounts to obtain momentum-dependent form factors that carry the dynamical content of the interaction, though a rigorous determination in the framework of a Lagrangian formalism such as the standard model has not yet been achieved. From a field theoretical point of view, the goal is to evaluate the Green's functions from the quantum action functional but, in practice, this is a poorly known procedure overcome by many uncertainties that arise because of hadronization and analytic continuation. As a consequence we rely on the construction of form factors from guiding principles such as analyticity, symmetries of the standard model, or model-dependent assumptions of dynamical content (vector meson dominance, factorization, duality and so forth). A more phenomenological approach consists in fitting ad hoc parametrizations with experimental data.

Hadronic low-energy phenomenology far below the resonance region $\left(E \ll M_{\rho}\right)$ is successfully described in the framework of chiral perturbation theory $(\chi \mathrm{PT})[1-3]$, the effective action of quantum chromodynamics (QCD) at low energies. However a similar tool in the resonance region, typically $E \sim 1 \mathrm{GeV}$, is still lacking, and has become a focus of interest in the last years. Data on $\pi \pi \rightarrow \pi \pi$, the vector pion form factor, hadronic $\tau$ decays and other processes have prompted the activity on the theoretical side.

Following the phenomenological Lagrangian ideas of Ref. [4], the inclusion of meson resonances in an effective theory was addressed in Ref. [5] by introducing the leading resonance chiral effective theory of QCD. It has to be emphasized that this is a model-independent framework that provides [6], upon integration of the spin-1 meson degrees of freedom, the same $\mathcal{O}\left(p^{4}\right) \chi \mathrm{PT}$ Lagrangian independently of the definition of the spin-1 fields, a nontrivial feature.

The problem we wish to study comes from the obvious fact that, in the resonance region, the width of the resonances cannot be neglected and one needs to regularize the poles in a proper way. Here we address the construction of the offshell width of meson resonances from the resonance chiral effective theory of QCD. We will focus, owing to its relevance and simplicity, in the off-shell width of the $\rho^{0}$. As it is well known the $\rho$ meson plays a crucial role in the dynamics of hadron processes at low energies and therefore its properties, like mass and width, have been thoroughly studied in many different frameworks $[7,8]$ usually related with the pion form factor. Notwithstanding most of these studies rely on modelizations of the interaction that include assumptions not justified from the standard model.

In Ref. [8] it was pointed out that one could consider the evaluation of the off-shell $\rho$ width from the effective theory of QCD at low energies that includes the resonances and Goldstone bosons explicitly. Here we carry out in detail such a procedure. We study two physical observables: the vector pion form factor and the $\pi^{+} \pi^{-} \rightarrow \pi^{+} \pi^{-}$amplitude with $J$ $=1$ in the $s$ channel. We construct a Dyson-Schwinger-like equation and we show that, in both observables, it gives the same imaginary part for the $\rho^{0}$ pole.

When considering off-shell processes one might worry about the fact that different redefinitions of the fields give different results. We have shown that the result of our procedure does not depend on the definition of the $\rho^{0}$ field. One of the conclusions of Ref. [6] was that, as different redefinitions of the spin-1 fields give different Lagrangians, interaction vertices are dependent on the formulation and, therefore, the Feynman diagrams contributing to a process are formulation-dependent. We will have to take this fact into account when considering which diagrams have to be accounted for in the Dyson-Schwinger series. As a result this is not going to be reduced to evaluate resonance self-energies only.

We will proceed then by proposing a definition of the off-shell width of the vector meson resonances as the imaginary part of the pole generated through the two-point vector 
current correlator, where only those diagrams with an absorptive part in the $s$ channel are included. This definition is shown to satisfy the crucial requirements of analyticity, unitarity, chiral symmetry and asymptotic behavior ruled by QCD. Again the result is shown to be independent of the spin-1 field formulation.

In Sec. II we study the resummation that regulates the pole of the $\rho^{0}$ in the vector pion form factor and the elastic scattering process $\pi^{+} \pi^{-} \rightarrow \pi^{+} \pi^{-}$. Then in Sec. III we propose a general definition of the off-shell width of resonances. Section IV is devoted to provide the rationale for the independence of our results from the spin-1 meson formulation. Our conclusions are pointed out in Sec. V.

\section{THE POLE OF RESONANCES IN PHYSICAL OBSERVABLES}

The position of the pole of the bare propagator for stable particles gets shifted when interactions are switched on. In the usual perturbative treatment of the interaction, a pole has to be achieved through a resummation procedure of higher orders. The well known solution of the Dyson-Schwinger equation for the dressed propagators, obtained through the evaluation of self-energy Feynman diagrams, hides the fact that the definition of the resummation, that is, which are the contributions and how one has to proceed, is not free of ambiguities. These are lessened if one needs to impose more restrictions on the result, like gauge invariance (see [9] and references therein). However, hadron processes at low energies are described by an effective action where color SU(3) gauge invariance is not explicit. At the $1 \mathrm{GeV}$ scale we are driven by two all-important features: chiral symmetry and the asymptotic behavior of form factors ruled by QCD. Obviously basic principles like analyticity and unitarity must also be satisfied.

Chiral symmetry has a long history as a powerful tool to describe low-energy hadrodynamics [10]. One of its main aspects is that it is a spontaneously broken symmetry that requires the existence of Goldstone bosons to be identified with the octet of lightest pseudoscalars. Chiral perturbation theory is the effective action of low-energy QCD [1-3] that, in the $S U(3)_{L} \otimes S U(3)_{R}$ version, involves only the octet of pions, kaons and $\eta$ mesons and describes strong interactions at $E \ll M_{\rho}$. At the $1 \mathrm{GeV}$ energy region the inclusion of the lightest resonances as explicit degrees of freedom is required.

The resonance chiral effective theory with three flavors and only including vector meson resonances is given, at the lowest chiral order, by [5]

$$
\mathcal{L}_{\chi V}=\mathcal{L}_{\chi}^{(2)}+\mathcal{L}_{K V}+\mathcal{L}_{V}^{(2)} .
$$

Here $\mathcal{L}_{\chi}^{(2)}$ is the $\mathcal{O}\left(p^{2}\right)$ chiral Lagrangian

$$
\mathcal{L}_{\chi}^{(2)}=\frac{F^{2}}{4}\left\langle u_{\mu} u^{\mu}+\chi_{+}\right\rangle
$$

where $F$ is the pion decay constant $(F \approx 92.4 \mathrm{MeV})$,

$$
u_{\mu}=i\left[u^{\dagger}\left(\partial_{\mu}-i r_{\mu}\right) u-u\left(\partial_{\mu}-i l_{\mu}\right) u^{\dagger}\right],
$$

$$
\begin{gathered}
\chi_{+}=u^{\dagger} \chi u^{\dagger}+u \chi^{\dagger} u, \\
u=\exp \left(\frac{i}{\sqrt{2} F} \Pi\right),
\end{gathered}
$$

and $\Pi$ is the usual representation of the Goldstone fields

$$
\Pi=\left(\begin{array}{ccc}
\frac{\pi^{0}}{\sqrt{2}}+\frac{\eta_{8}}{\sqrt{6}} & \pi^{+} & K^{+} \\
\pi^{-} & -\frac{\pi^{0}}{\sqrt{2}}+\frac{\eta_{8}}{\sqrt{6}} & K^{0} \\
K^{-} & \bar{K}^{0} & -\frac{2}{\sqrt{6}} \eta_{8}
\end{array}\right) .
$$

In Eq. (2), $\langle A\rangle$ stands for a trace in the flavor space. $\mathcal{L}_{\chi}^{(2)}$ has a $S U(3)_{L} \otimes S U(3)_{R}$ chiral gauge symmetry supported by the external fields $l_{\mu}, r_{\mu}$, and $\chi$.

In Eq. (1), $\mathcal{L}_{K V}$ is the kinetic Lagrangian of vector mesons and $\mathcal{L}_{V}^{(2)}$ describes the chiral couplings of vector mesons to the Goldstone fields and external currents at the lowest order,

$$
\mathcal{L}_{V}^{(2)}=\frac{F_{V}}{2 \sqrt{2}}\left\langle V_{\mu \nu} f_{+}^{\mu \nu}\right\rangle+i \frac{G_{V}}{\sqrt{2}}\left\langle V_{\mu \nu} u^{\mu} u^{\nu}\right\rangle,
$$

where $f_{+}^{\mu \nu}=u F_{L}^{\mu \nu} u^{\dagger}+u^{\dagger} F_{R}^{\mu \nu} u$, with $F_{L, R}^{\mu \nu}$ the field strength tensors of the left and right external currents $l_{\mu}$ and $r_{\mu} . V_{\mu \nu}$ denotes the octet of the lightest vector mesons, in the antisymmetric formulation $[2,5,11]$, with a flavor content analogous to $\Pi$ in Eq. (4). The effective couplings $F_{V}$ and $G_{V}$ can be determined from the decays $\rho^{0} \rightarrow e^{+} e^{-}$and $\rho^{0} \rightarrow \pi^{+} \pi^{-}$ respectively. Notice that only linear terms in the vector fields have been considered in $\mathcal{L}_{V}^{(2)}$.

Assuming unsubtracted dispersion relations for the pion and axial form factors one gets two constraints [6] among the couplings $F, F_{V}$, and $G_{V}$ :

$$
F_{V} G_{V}=F^{2}, \quad F_{V}=2 G_{V}
$$

which are reasonably well satisfied phenomenologically. We will enforce those constraints in our analysis.

As thoroughly studied in Ref. [6] the use of the antisymmetric formalism to describe spin-1 mesons simplifies the structure of the effective action at the lowest chiral order in the even-intrinsic parity sector. If we use the Proca formulation of the vector fields instead, we need to consider the $\mathcal{O}\left(p^{4}\right)$ chiral Lagrangian of Gasser and Leutwyler [3], with appropriate $L_{i}$ coefficients, in order to satisfy the shortdistance QCD constraints. We will comment later on about the independence of our results on this particular choice.

The bare propagator of the vector mesons, in the antisymmetric formulation, is given by 

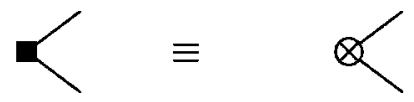

$+$

(a)
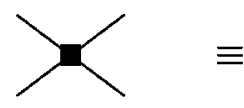
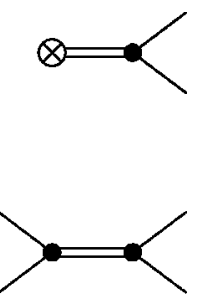

(s-channel)

(b)

FIG. 1. Effective vertices contributing to vector transitions in the $s$ channel that are relevant for the pion form factor and elastic $\pi \pi$ scattering. The crossed circle stands for an external vector current insertion. A double line indicates the $\rho^{0}$ meson and single ones the pseudoscalars. Local vertices on the right-hand side are provided, at leading order, by $\mathcal{L}_{\chi}^{(2)}$.

$$
\begin{aligned}
\left\langle 0\left|T\left\{V_{\mu \nu}(x), V_{\rho \sigma}(y)\right\}\right| 0\right\rangle & \\
= & \frac{i}{M_{V}^{2}} \int \frac{d^{4} q}{(2 \pi)^{4}} \frac{e^{-i q(x-y)}}{M_{V}^{2}-q^{2}-i \varepsilon}\left[M_{V}^{2} \Omega_{\mu \nu \rho \sigma}^{L}+\Omega_{\mu \nu \rho \sigma}^{T}\right],
\end{aligned}
$$

with

$$
\begin{aligned}
& \Omega_{\mu \nu \rho \sigma}^{L} \doteq g_{\mu \rho} g_{\nu \sigma}-g_{\mu \sigma} g_{\nu \rho}, \\
& \Omega_{\mu \nu \rho \sigma}^{T} \doteq g_{\mu \rho} q_{\nu} q_{\sigma}-g_{\rho \nu} q_{\sigma} q_{\mu}-q^{2} g_{\mu \rho} g_{\nu \sigma}-(\rho \leftrightarrow \sigma),
\end{aligned}
$$

that satisfies $q^{\mu} \Omega_{\mu \nu \rho \sigma}^{T}=0$. Chiral symmetry requires that the interaction between the vector mesons and pseudoscalars or external currents is a derivative coupling, as shown in $\mathcal{L}_{V}^{(2)}$. Consequently, when the vector-meson propagator connects with only one external current or two pseudoscalars, the transverse part $\Omega_{\mu \nu \rho \sigma}^{T}$ does not contribute.

As commented above, a Lagrangian density that includes the Goldstone bosons and spin-1 resonances is not unique but depends on the definitions of the fields, whereas the observable physical quantities should be independent of them. If we want to construct the dressed propagator of the $\rho^{0}$ meson we should consider, for a definite intermediate state, all the contributions carrying the appropriate quantum numbers. The first cut, in the $\rho^{0}$ case, is a two-pseudoscalar absorptive contribution that happens to saturate its width. Here we will take into account the two-particle absorptive contributions only; higher multiplicity intermediate states being suppressed by phase space and ordinary chiral counting. The effective vertices, that will contribute to the observables we are interested in, are those corresponding to an external vector current coupled to two pseudoscalar legs, and to a vector transition in the $s$ channel contributing to the four pseudoscalar vertex. These transitions are not only diagrammatically driven by the $\rho$ propagator, but also through local contributions that have to be included. The construction of the effective vertices goes as sketched in Fig. 1 where, at the leading order, the local vertices on the right-hand side of the equivalence are provided by the $\mathcal{O}\left(p^{2}\right)$ chiral Lagrangian $\mathcal{L}_{\chi}^{(2)}$ in Eq. (2). The diagrams contributing to physical ob-

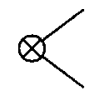

(a)

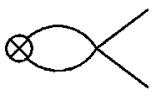

(d)

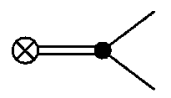

(b)

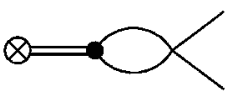

(e)

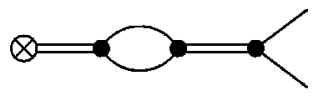

(c)

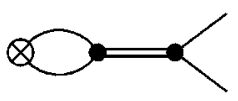

(f)
FIG. 2. Diagrams contributing to the vector pion form factor up to one loop in the resonance chiral Lagrangian given by $\mathcal{L}_{\chi V}$ that have an absorptive part in the $s$ channel. The lines and symbols stand for as in Fig. 1.

servables will be constructed taking into account all possible combinations of these two effective vertices.

Let us start with the vector pion form factor $F_{V}\left(q^{2}\right)$ defined by

$$
\left\langle\pi^{+}\left(p^{\prime}\right)\left|v_{\mu}^{3}\right| \pi^{+}(p)\right\rangle=\left(p+p^{\prime}\right)_{\mu} F_{V}\left(q^{2}\right),
$$

where $q^{2}=\left(p-p^{\prime}\right)^{2}$ and $v_{\mu}^{3}$ is the third $S U(3)$ component of the vector current $v_{\mu}=\left(l_{\mu}+r_{\mu}\right) / 2 . F_{V}\left(q^{2}\right)$ is dominated by the contribution of the $\rho^{0}$ meson and has thoroughly been studied in Ref. [8] up to $E \sim 1.5 \mathrm{GeV}$. Clearly, one cannot describe $F_{V}\left(q^{2}\right)$ in that region of energy using the bare $\rho^{0}$ propagator of Eq. (7); the width of the resonance has to be introduced to regulate the pole of the propagator.

We propose then to construct a Dyson-Schwinger-like equation through a perturbative loop expansion. According to our previous discussion, at tree level one has to take into account the amplitudes provided by Figs. 2(a) and 2(b), i.e., the effective vertex in Fig. 1(a). The next step is to consider one-loop corrections. We are only interested in those contributions with absorptive parts in the $s$ channel. They are generated by inserting a pseudoscalar loop using the two effective vertices in Fig. 1 which leads to the four contributions in Figs. 2(c), 2(d), 2(e), and 2(f). In this way we have proceeded up to two loops. The resulting infinite series happens to be geometric and its resummation gives

$$
F_{V}\left(q^{2}\right)=\frac{M_{V}^{2}}{M_{V}^{2}\left[1+2 \frac{q^{2}}{F^{2}} \operatorname{Re} \overline{B_{22}}\right]-q^{2}-i M_{V} \Gamma_{\rho}\left(q^{2}\right)},
$$

where $M_{V}$ is the mass of the octet of vector mesons in the chiral limit, $\overline{B_{22}} \equiv B_{22}\left[q^{2}, m_{\pi}^{2}, m_{\pi}^{2}\right]+\frac{1}{2} B_{22}\left[q^{2}, m_{K}^{2}, m_{K}^{2}\right]$ and the $B_{22}\left[q^{2}, m^{2}, m^{2}\right]$ function is given in the Appendix. The width of the $\rho^{0}$ meson $\Gamma_{\rho}\left(q^{2}\right)$ is given by

$$
\begin{aligned}
\Gamma_{\rho}\left(q^{2}\right) & =-2 M_{V} \frac{q^{2}}{F^{2}} \operatorname{Im} \overline{B_{22}} \\
& =\frac{M_{V} q^{2}}{96 \pi F^{2}}\left[\sigma_{\pi}^{3} \theta\left(q^{2}-4 m_{\pi}^{2}\right)+\frac{1}{2} \sigma_{K}^{3} \theta\left(q^{2}-4 m_{K}^{2}\right)\right],
\end{aligned}
$$


where $\sigma_{P}=\sqrt{1-4 m_{P}^{2} / q^{2}}$.

The real part of the pole of $F_{V}\left(q^{2}\right)$ in Eq. (10) needs still to be regulated through the wave function and mass renormalization of the vector field. The local part of $\operatorname{Re} B_{22}$ can be fixed in this case by matching the result in Eq. (10) with the well known expression of $F_{V}\left(q^{2}\right)$ at one loop in $\chi \mathrm{PT}[8,12]$. One gets then

$$
\begin{aligned}
B_{22} & {\left.\left[q^{2}, m_{P}^{2}, m_{P}^{2}\right]\right|_{F_{V}} } \\
& =\frac{1}{192 \pi^{2}}\left[\ln \left(\frac{m_{P}^{2}}{M_{V}^{2}}\right)+8 \frac{m_{P}^{2}}{q^{2}}-\frac{5}{3}+\sigma_{P}^{3} \ln \left(\frac{\sigma_{P}+1}{\sigma_{P}-1}\right)\right] .
\end{aligned}
$$

We have used the standard vector meson dominance value [5] of the relevant $\mathcal{O}\left(p^{4}\right)$ chiral coupling, $L_{9}\left(M_{V}^{2}\right)$ $=F^{2} / 2 M_{V}^{2}$, which agrees very well with its phenomenologically extracted value.

To one-loop accuracy, Eq. (10) agrees by construction with the Omnès resummation of chiral logarithms performed in Ref. [8]. However, and as pointed out in Ref. [13], the Omnès resummation also reproduces the contribution of double chiral logarithms, while our one-loop resummation does not [14]. This is not surprising, since our result in Eq. (10) has been obtained by considering not all possible diagrams but only those which are driven by the $\rho^{0}$ resonance and contribute to its width. It would be interesting to perform a detailed study of both resummations at the next chiral order and compare them with the known two-loop $\chi \mathrm{PT}$ results [15].

An analogous procedure can be applied to the study of elastic $\pi \pi$ scattering. For definiteness we take the $J=1$ transition in the $s$-channel amplitude of $\pi^{+} \pi^{-} \rightarrow \pi^{+} \pi^{-}$. This channel is again dominated by the $\rho^{0}$ meson and we can proceed to construct a Dyson-Schwinger equation as in the case of the vector pion form factor. Thus, we consider analogous diagrams to those in Fig. 2, substituting the external vector current insertions by two pion legs, according to all the possible contributions of the effective vertices in Fig. 1. By projecting the $p$ wave (that corresponds to the $J=1$ contribution) we find again a geometric series, which can be resummed to give

$$
\begin{aligned}
\left.\mathcal{A}\left(\pi^{+} \pi^{-} \rightarrow \pi^{+} \pi^{-}\right)\right|_{J=1} & \\
= & \frac{-i}{2 F^{2}}(u-t) \\
& \times \frac{M_{V}^{2}}{M_{V}^{2}\left[1+2 \frac{q^{2}}{F^{2}} \operatorname{Re} \overline{B_{22}}\right]-q^{2}-i M_{V} \Gamma_{\rho}\left(q^{2}\right)},
\end{aligned}
$$

where $u$ and $t$ are the usual Mandelstam variables $\left(q^{2}=s\right)$. We see that the pole of the amplitude coincides with the one we got for the vector pion form factor and, therefore, gives the same width of the $\rho^{0}$ meson. Contrary to the pion form

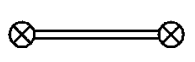

(a)

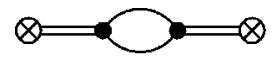

(b)

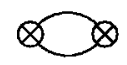

(c)

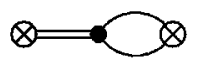

(d)

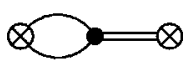

(e)
FIG. 3. Diagrams contributing to the vector-vector correlator $\Pi_{\mu \nu}^{33}$ up to one loop. The lines and symbols stand for as in Fig. 1.

factor case, here it is not possible to fix the polynomial part of the $\operatorname{Re} B_{22}$ function in a simple way, because a proper matching with the chiral low-energy expansion requires us to take into account $p$-wave contributions in the $t$ and $u$ channels, which are not accounted for in our result of Eq. (13).

In the next section we will introduce a definition of the off-shell width of spin-1 resonances that is consistent with our previous results.

\section{THE DEFINITION OF A HADRONIC OFF-SHELL $\rho^{0}$ WIDTH}

We propose to define the spin- 1 meson width as the imaginary part of the pole generated by resumming those diagrams, with an absorptive part in the $s$ channel, that contribute to the two-point function of the corresponding vector current. That is, the pole of

$$
\Pi_{\mu \nu}^{j k}=i \int d^{4} x e^{i q x}\left\langle 0\left|T\left[V_{\mu}^{j}(x) V_{\nu}^{k}(0)\right]\right| 0\right\rangle,
$$

with

$$
V_{\mu}^{j}=\frac{\delta S_{\chi V}}{\delta v_{j}^{\mu}},
$$

where $S_{\chi V}$ is the action associated to $\mathcal{L}_{\chi V}$.

The $\rho^{0}$ quantum numbers correspond to $j=k=3$. Lorentz covariance and current conservation allow us to define an invariant function of $q^{2}$ through

$$
\begin{aligned}
\Pi_{\mu \nu}^{33} & =\left(q^{2} g_{\mu \nu}-q_{\mu} q_{\nu}\right) \Pi^{\rho}\left(q^{2}\right), \\
\Pi^{\rho}\left(q^{2}\right) & =\Pi_{(0)}^{\rho}+\Pi_{(1)}^{\rho}+\Pi_{(2)}^{\rho}+\cdots,
\end{aligned}
$$

where $\Pi_{(0)}^{\rho}$ corresponds to the tree level contribution of Fig. $3(\mathrm{a}), \Pi_{(1)}^{\rho}$ to the one-loop amplitudes and so forth. Up to one loop, and considering again the two-particle absorptive contributions only, all the diagrams generated by the effective vertices in Fig. 1 are shown in Fig. 3. We find, in the isospin limit,

$$
\begin{aligned}
& \Pi_{(0)}^{\rho}=\frac{F_{V}^{2}}{M_{V}^{2}-q^{2}}, \\
& \Pi_{(1)}^{\rho}=\Pi_{(0)}^{\rho}\left[-\frac{M_{V}^{2}}{F_{V}^{2}} \frac{M_{V}^{2}}{M_{V}^{2}-q^{2}} 4 \overline{B_{22}}\right] .
\end{aligned}
$$




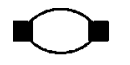

(a)

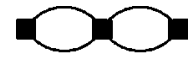

(b)
FIG. 4. One- and two-loop diagrams leading to $\Pi_{(1)}^{\rho}$ (a) and $\Pi_{(2)}^{\rho}$ (b). The effective squared vertices are given in Fig. 1.

QCD predicts that two-point spectral functions of vector currents go to a constant value as $q^{2} \rightarrow \infty$ [16]. The loop diagram in Fig. 3(b) behaves also as a constant for large values of $q^{2}$, which is against the expectations because it corresponds to only one of an infinite number of possible intermediate states. In order to satisfy the QCD prediction, one would foresee that all the individual (positive) contributions from the different intermediate states should vanish in the limit $q^{2} \rightarrow \infty$. Indeed, this is achieved in our case when diagrams in Figs. 3(c), 3(d), and 3(e) are added.

Our result for $\Pi_{(1)}^{\rho}$ corresponds to a single one-loop diagram with two effective vertices, as shown in Fig. 4(a). It vanishes as $q^{2} \rightarrow \infty$, as QCD requires, and this fact happens at every higher order when all possible diagrams with absorptive contributions in the $s$ channel (and not just selfenergies) are included.

We proceed to evaluate $\Pi_{(2)}^{\rho}$, that is, the contribution of two-loop diagrams that arise from $\mathcal{L}_{\chi V}$ with absorptive contributions in the $s$ channel. These diagrams can be easily constructed by iterating in all possible ways the one-loop diagrams shown in Fig. 3; they correspond to the single twoloop diagram with three effective vertices shown in Fig. 4(b). We obtain

$$
\Pi_{(2)}^{\rho}=\Pi_{(1)}^{\rho}\left[-\frac{q^{2}}{F_{V}^{2}} \frac{M_{V}^{2}}{M_{V}^{2}-q^{2}} 4 \overline{B_{22}}\right]
$$

A watchful look to the evaluation (and a check up to threeloops) makes us to conclude that the invariant two-point function $\Pi^{\rho}\left(q^{2}\right)$, generated by resumming effective loop diagrams with an absorptive amplitude in the $s$ channel (as those in Fig. 4), is perturbatively given by

$$
\begin{aligned}
\Pi^{\rho}\left(q^{2}\right) & =\Pi_{(0)}^{\rho}+\Pi_{(1)}^{\rho} \sum_{n=0}^{\infty}\left[-\frac{q^{2}}{F_{V}^{2}} \frac{M_{V}^{2}}{M_{V}^{2}-q^{2}} 4 \overline{B_{22}}\right]^{n} \\
& =\Pi_{(0)}^{\rho}\left[1+\omega \sum_{n=0}^{\infty}\left(\frac{q^{2}}{M_{V}^{2}} \omega\right)^{n}\right]
\end{aligned}
$$

where

$$
\omega=-\frac{M_{V}^{2}}{F_{V}^{2}} \frac{M_{V}^{2}}{M_{V}^{2}-q^{2}} 4 \overline{B_{22}} .
$$

Now, resumming, using that $F_{V}^{2}=2 F^{2}$ [see Eq. (6)], and substituting the expression of $\omega$, we finally get

$$
\begin{aligned}
\Pi^{\rho}\left(q^{2}\right)= & \frac{2 F^{2}}{M_{V}^{2}\left[1+2 \frac{q^{2}}{F^{2}} \operatorname{Re} \overline{B_{22}}\right]-q^{2}-i M_{V} \Gamma_{\rho}\left(q^{2}\right)} \\
& \times\left[1-2 \frac{M_{V}^{2}}{F^{2}} \overline{B_{22}}\right],
\end{aligned}
$$

where the off-shell $\rho^{0}$ width $\Gamma_{\rho}\left(q^{2}\right)$ is given by Eq. (11).

We emphasize that our only concern with the result of $\Pi^{\rho}\left(q^{2}\right)$ in Eq. (22) is the reconstruction of the imaginary part of the pole. Our evaluation of the two-point function vector-vector correlator is far from complete, since we have considered only those diagrams with absorptive contributions in the $s$ channel. ${ }^{1}$ The only significative result of $\Pi^{\rho}\left(q^{2}\right)$ is its imaginary part. The residue in $\Pi^{\rho}\left(q^{2}\right)$ deserves a further comment. While it carries an imaginary piece, this result is proper as far as it satisfies the required unitarity condition

$$
\begin{aligned}
\operatorname{Im} \Pi^{\rho}\left(q^{2}\right)= & \frac{1}{48 \pi}\left[\sigma_{\pi}^{3} \theta\left(q^{2}-4 m_{\pi}^{2}\right)+\frac{1}{2} \sigma_{K}^{3} \theta\left(q^{2}-4 m_{K}^{2}\right)\right] \\
& \times\left|F_{V}\left(q^{2}\right)\right|^{2}
\end{aligned}
$$

with $F_{V}\left(q^{2}\right)$ given by our result in Eq. (10). This shows the consistency of our resummation procedure.

\section{INDEPENDENCE OF THE SPIN-1 FIELD DEFINITION}

In Ref. [6] it was shown that, at $\mathcal{O}\left(p^{4}\right)$ in the chiral expansion, sensible redefinitions of the spin-1 fields give the same results for physical low-energy observables. The equivalence between redefinitions may require the presence of local terms that have to be added in order to satisfy the short-distance QCD constraints. In that reference it was concluded that, at $\mathcal{O}\left(p^{4}\right)$, the antisymmetric formulation for spin-1 meson resonances is the only one that does not require the presence of such local contributions and is, therefore, the simplest one.

However, a resummation of the two-point vector-vector correlator when vectors are active degrees of freedom has no defined chiral counting. Therefore, one might worry that $\Gamma_{\rho}\left(q^{2}\right)$ could depend on the field formulation used to describe the vector mesons.

In order to see the independence of our results on the spin-1 field formulation, it is enough to realize that the effective vertices defined in Fig. 1 are universal. Different theoretical descriptions of the vector (or axial-vector) meson degrees of freedom lead to resonance-exchange contributions which differ by local terms. Since the physical amplitudes are constrained to satisfy the appropriate QCD behavior at

\footnotetext{
${ }^{1}$ In fact, those diagrams where the vector fields are not explicit [such as that in Fig. 3(c)] give rise to a dispersive divergent piece proportional to $q_{\mu} q_{\nu}$ that we disregard. It is also clear from our result in Eq. (22) that the real part of the pole needs regularization.
} 
large momenta, this difference is necessarily compensated by explicit local terms (with fixed couplings) [6]. Including those local terms in the local vertices of Fig. 1, the resulting effective vertices are formulation independent.

Since our resummation has been based on these effective vertices, the universality of our result follows.

Our discussion has been carried out for a two-particle cut, which is the most important contribution for the $\rho^{0}$ and other vector mesons. A similar procedure could be applied to higher multiplicity intermediate states by constructing the relevant effective vertices analogous to those in Fig. 1. Although technically much more involved, its study would be necessary to evaluate the width of other resonances such as the $\omega(872)$ or the axial-vector mesons.

\section{CONCLUSIONS}

In this work we have studied the off-shell width of spin-1 meson resonances in a model-independent framework provided by the resonance chiral effective theory of QCD at low energies. We have performed resummations for the vector pion form factor and the $\pi \pi \rightarrow \pi \pi(J=1)$ amplitude, showing that they provide the same structure for the pole of the $\rho^{0}$ vector meson. In both cases the resummations correspond to geometric Dyson-Schwinger-like series that include only diagrams with absorptive contributions in the $s$ channel.

We have defined the width of spin-1 meson resonances as given by the imaginary part of the pole generated by resumming loop diagrams, in the two-point correlator of vector or axial-vector currents of the resonance chiral Lagrangian, that have absorptive contributions in the $s$ channel. The width generated in this way satisfies the requirements of analyticity, unitarity, chiral symmetry, and the correct asymptotic behavior as prescribed by QCD.

We have applied this procedure to evaluate the off-shell width of the $\rho^{0}$ meson and we have worked out in detail the result. Moreover, we have shown that this definition is independent of the formulation employed to describe the vector meson fields. Hence our procedure can be applied straightaway to evaluate the widths of other spin-1 resonances.

\section{ACKNOWLEDGMENTS}

This work has been supported in part by TMR, EC Contract No. ERB FMRX-CT98-0169 and by CICYT (Spain) under grants PB97-1261 and AEN99-0692. D.G.D. acknowledges the warm hospitality given by the Departament de Física Teorica of the University of Valencia, and the financial support provided by Fundación Antorchas, Argentina. J.P. wishes to thank F.J. Botella for interesting discussions on the subject of this paper.

\section{APPENDIX}

The function $B_{22}\left[q^{2}, m^{2}, m^{2}\right]$ used in the text is defined through

$$
\begin{gathered}
\int \frac{d^{D} l}{i(2 \pi)^{D}} \frac{l_{\mu} l_{\nu}}{\left[l^{2}-m^{2}\right]\left[(l-q)^{2}-m^{2}\right]} \\
\equiv q_{\mu} q_{\nu} B_{21}+q^{2} g_{\mu \nu} B_{22},
\end{gathered}
$$

as

$$
\begin{aligned}
B_{22}\left[q^{2}, m^{2}, m^{2}\right]= & \frac{1}{192 \pi^{2}}\left[\left(1-6 \frac{m^{2}}{q^{2}}\right)\left[\lambda_{\infty}+\ln \left(\frac{m^{2}}{\mu^{2}}\right)\right]\right. \\
& \left.+8 \frac{m^{2}}{q^{2}}-\frac{5}{3}+\sigma^{3} \ln \left(\frac{\sigma+1}{\sigma-1}\right)\right],
\end{aligned}
$$

where $\quad \sigma=\sqrt{1-4 m^{2} / q^{2}} \quad$ and $\quad \lambda_{\infty}=[2 /(D-4)] \mu^{D-4}$ $-\left[\Gamma^{\prime}(1)+\ln (4 \pi)+1\right]$.
[1] S. Weinberg, Physica A 96, 327 (1979).

[2] J. Gasser and H. Leutwyler, Ann. Phys. (N.Y.) 158, 142 (1984).

[3] J. Gasser and H. Leutwyler, Nucl. Phys. B250, 465 (1985).

[4] S. Coleman, J. Wess, and B. Zumino, Phys. Rev. 177, 2239 (1969); C.G. Callan, S. Coleman, J. Wess, and B. Zumino, ibid. 177, 2247 (1969).

[5] G. Ecker, J. Gasser, A. Pich, and E. de Rafael, Nucl. Phys. B321, 311 (1989).

[6] G. Ecker, J. Gasser, H. Leutwyler, A. Pich, and E. de Rafael, Phys. Lett. B 223, 425 (1989).

[7] J. Pišút and M. Roos, Nucl. Phys. B6, 325 (1968); G.J. Gounaris and J.J. Sakurai, Phys. Rev. Lett. 21, 244 (1968); M.B. Einhorn, Phys. Rev. 185, 1960 (1969); M. Roos, Nucl. Phys. B97, 165 (1975); A. Bernicha, G. López Castro, and J. Pestieau, Phys. Rev. D 50, 4454 (1994); F. Klingl, N. Kaiser, and W. Weise, Z. Phys. A 356, 193 (1996); M. Roos, Acta
Phys. Slov. 49, vii (1999); M. Benayoun et al., Eur. Phys. J. C 2, 269 (1998); ALEPH Collaboration, R. Barate et al., Z. Phys. C 76, 15 (1997); CLEO Collaboration, S. Anderson et al., Phys. Rev. D 61, 112002 (2000).

[8] F. Guerrero and A. Pich, Phys. Lett. B 412, 382 (1997).

[9] J. Papavassiliou and A. Pilaftsis, Phys. Rev. D 54, 5315 (1996).

[10] G. Ecker, Prog. Part. Nucl. Phys. 35, 1 (1995); A. Pich, Rep. Prog. Phys. 58, 563 (1995).

[11] E. Kyriakopoulos, Phys. Rev. 183, 1318 (1969).

[12] J. Gasser and H. Leutwyler, Nucl. Phys. B250, 517 (1985).

[13] J. Gasser and Ulf-G. Meissner, Nucl. Phys. B357, 90 (1991).

[14] F. Guerrero, Phys. Rev. D 57, 4136 (1998).

[15] J. Bijnens, G. Colangelo, and P. Talavera, J. High Energy Phys. 05, 014 (1998).

[16] E.G. Floratos, S. Narison, and E. de Rafael, Nucl. Phys. B155, 115 (1979). 